\title{
Second-Order Hydrodynamics in Next-to-Leading-Order QCD
}

\author{
Jacopo Ghiglieri, ${ }^{1}$ Guy D. Moore, ${ }^{2}$ and Derek Teaney ${ }^{3}$ \\ ${ }^{1}$ Theoretical Physics Department, CERN, Geneva, CH-1211, Switzerland \\ ${ }^{2}$ Institut für Kernphysik, Technische Universität Darmstadt Schlossgartenstraße 2, D-64289 Darmstadt, Germany \\ ${ }^{3}$ Department of Physics and Astronomy, Stony Brook University, Stony Brook, New York 11794-3800, USA
}

(Received 15 May 2018; published 31 July 2018)

\begin{abstract}
We compute the hydrodynamic relaxation times $\tau_{\pi}$ and $\tau_{j}$ for hot QCD at next-to-leading order in the coupling with kinetic theory. We show that certain dimensionless ratios of second-order to first-order transport coefficients obey bounds which apply whenever a kinetic theory description is possible; the computed values lie somewhat above these bounds. Strongly coupled theories with holographic duals strongly violate these bounds, highlighting their distance from a quasiparticle description.
\end{abstract}

DOI: $10.1103 /$ PhysRevLett.121.052302

Introduction.-The quark-gluon plasma (QGP) produced at RHIC [1,2] and the LHC [3-6] appears to be an excellent fluid. Despite the small system size, viscous hydrodynamics does a good job describing many collective properties, spectra, and correlations [7,8]. To be causal and stable $[9,10]$, such treatments must work to second order in the gradient expansion, requiring many more coefficients than the celebrated shear viscosity to entropy ratio $\eta / s$. In particular, a treatment of collective flow requires not only the shear viscosity $\eta$ but also the shear relaxation rate $\tau_{\pi}$, and baryon-number diffusion needs not just a diffusion coefficient $D_{q}$ but also a diffusive relaxation time $\tau_{j}$.

We would like to use experiments to constrain the properties of the QGP such as $\eta / s$, but the necessity to include higher-order coefficients could lead to a proliferation of fitting parameters. So one often assumes that the coefficients follow some simple relations, such as $\tau_{\pi}=$ $K \eta /(\epsilon+\mathcal{P})$, with $(\epsilon+\mathcal{P})$ the enthalpy density and $K$ a constant which we draw from some microscopic theory of relativistic plasmas. For instance, York and Moore showed that weakly coupled massless QCD treated to leading order (LO) in the gauge coupling yields $5<K<6$, nearly independent of coupling strength [11], while Baier et al. find that strongly coupled $\mathcal{N}=4$ super-Yang-Mills (SYM) theory has $K \simeq 2.62$ [12].

Recently, we extended previous perturbative results for the shear viscosity and baryon-number diffusion of hot QCD from leading [13] to next-to-leading order (NLO) [14]; see Fig. 1. How does a NLO treatment change $K$ ? In this Letter, we will explore this issue. In addition to finding

Published by the American Physical Society under the terms of the Creative Commons Attribution 4.0 International license. Further distribution of this work must maintain attribution to the author(s) and the published article's title, journal citation, and DOI. Funded by SCOAP ${ }^{3}$. concrete results for $K$ and $\tau_{j} / D_{q}$, we will also show very general bounds on these dimensionless ratios which follow as soon as we state that a theory is well described by relativistic kinetic theory. These bounds are badly violated by strongly coupled theories with holographic duals, with the interesting implication that these theories are very far from having quasiparticle descriptions.

Definitions. - Let us start by defining the objects of our investigation. In the Landau-Lifshitz fluid rest frame, the stress tensor has the form

$$
\left\langle T^{i j}(x)\right\rangle=\delta^{i j}\langle\mathcal{P}\rangle+\pi^{i j},
$$

where the nonideal dissipative part can be gradient expanded. At first order,

$$
\pi_{1}^{i j}=-\eta\left(\nabla_{i} u^{j}+\nabla_{j} u^{i}-\frac{2}{3} \delta^{i j} \nabla_{l} u^{l}\right)-\zeta \delta^{i j} \nabla_{l} u^{l} .
$$

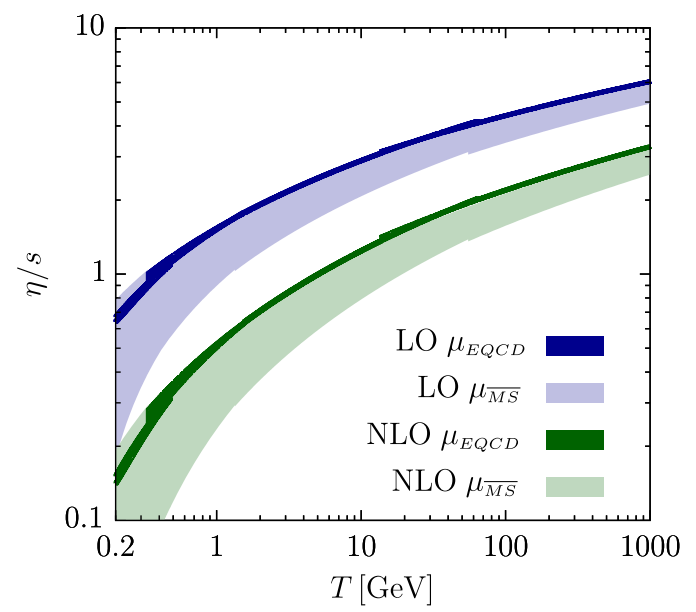

FIG. 1. $\eta / s$ of QCD as a function of the temperature at LO and NLO for the different choices of the running coupling detailed in Fig. 3 and in the text later on. Figure taken from Ref. [14]. 
We will concentrate on shear viscosity $\eta$ and not discuss bulk viscosity $\zeta$ further. At second order, the coefficients relevant for a conformal theory have been introduced in Ref. [12]. Here, we will only deal with second-order relaxation, whose coefficient $\tau_{\pi}$ is defined as $[15,16]$

$$
\tau_{\pi} \partial_{t} \pi^{i j}=\pi_{1}^{i j}-\pi^{i j} .
$$

When there are additional conserved global charges $Q_{\alpha}$ such as baryon or lepton number, the associated charge density $n_{\alpha} \equiv j^{0}$ and current density $\boldsymbol{j}$ satisfy, at first order in the gradients, a diffusion equation,

$$
\left\langle\dot{j}_{1}\right\rangle=-D_{\alpha} \nabla\left\langle n_{\alpha}\right\rangle=-k_{\mu_{\alpha}} \nabla \frac{\mu_{\alpha}}{T},
$$

where $D_{\alpha}$ is the diffusion coefficient. Here, we have rewritten the current with a gradient of the associated chemical potential $\mu_{\alpha}$. The associated transport coefficient $k_{\mu_{\alpha}}$ is related to $D_{\alpha}$ through the susceptibility $\chi_{\alpha}$ :

$$
D_{\alpha}=\frac{k_{\mu_{\alpha}}}{T \chi_{\alpha}}, \quad \chi_{\alpha} \equiv \frac{\partial n_{\alpha}}{\partial \mu_{\alpha}} .
$$

If we were to write Eq. (2) as a gradient of the charges $T^{0 j}=(\epsilon+\mathcal{P}) u^{j}$, we would naturally see that the associated relaxation coefficient is $\eta /(\epsilon+\mathcal{P})$. Analogous to Eq. (3), the second-order relaxation of $\boldsymbol{j}$ reads [18-20]

$$
\tau_{j} \partial_{t} \boldsymbol{j}=\boldsymbol{j}_{1}-\boldsymbol{j}
$$

In Refs. [13,21], it was shown how the first-order transport coefficients can be determined from a linearized kinetic theory. In Ref. [21], the collision operator defining the kinetic theory of QCD was determined at leading logarithmic accuracy, in Ref. [13] at LO, and in Refs. [14,22] at (almost) NLO. The kinetic theory expression for $\tau_{\pi}$ was derived in Ref. [11], leading to its LO determination.

First, we summarize the main findings of Refs. [11,13,21]. We start from a generic kinetic theory of the form

$$
\left(\frac{\partial}{\partial t}+\boldsymbol{v}_{\boldsymbol{p}} \cdot \frac{\partial}{\partial \boldsymbol{x}}\right) f^{a}(\boldsymbol{p}, \boldsymbol{x}, t)=-C_{a}[f],
$$

where $f^{a}(\boldsymbol{p}, \boldsymbol{x}, t)=d N^{a} / d^{3} \boldsymbol{x} d^{3} \boldsymbol{p}$ is the phase space distribution function for the excitation (gluon, quark, antiquark) of index $a$. If $u^{i}, \mu$ vary with space, then the localequilibrium form of $f^{a}$ does as well [23], $f_{0}^{a}=$ $\left[\exp \left(-\beta u^{\mu} P_{\mu}-q_{\alpha}^{a} \beta \mu\right) \mp 1\right]^{-1}$. The gradients on the lefthand side of Eq. (7), which we treat as perturbatively small, give rise to a source of departure from equilibrium $X^{i}=$ $\nabla_{i} \mu_{\alpha}$ for flavor diffusion $(\ell=1)$ and $X^{i j} \propto \pi_{1}^{i j} / \eta$ for shear $(\ell=2)$. This determines the linearized departure from equilibrium via a linearized version of Eq. (7),

$$
\mathcal{S}^{a}(\boldsymbol{p})=\left(\mathcal{C} f_{1}\right)^{a}(\boldsymbol{p}),
$$

where $\mathcal{S}^{a}=\beta q^{a} X_{i \cdots j} I_{i \cdots j}(\boldsymbol{p}) f_{0}^{a}\left[1 \pm f_{0}^{a}\right]$, with $q^{a}=q_{\alpha}^{a}$ for number diffusion and $p$ for shear. $f_{1}$ is the linearized departure from equilibrium, $f^{a}(\boldsymbol{p})=f_{0}^{a}(p)+f_{1}(\boldsymbol{p}) f_{0}[1 \pm$ $\left.f_{0}\right]$ and $I_{i \cdots j} \propto p_{i} \ldots p_{j}$ (see Refs. [13,21]). At linear order $f_{1}^{a} \propto X_{i \ldots j}$, allowing us to define the scalar function $\chi(p)[24]$

$$
f_{1}^{a}(\boldsymbol{p}) \equiv \beta^{2} X_{i \cdots j} I_{i \cdots j}(\hat{\boldsymbol{p}}) q^{a} \chi^{a}(p) .
$$

The linearized collision operator $\mathcal{C}$ is worked out in detail for the case of weakly coupled QCD in Ref. [13] at LO and in Ref. [14] at NLO.

General bounds.-To determine $\eta, D_{\alpha}, \tau_{\pi}$, and $\tau_{j}$, we will need to solve Eq. (8) to linear order in $f_{1}$ but to subleading order in gradients, which will depend in detail on the form of the collision operator. However, we can already make some generic statements about the solution, which will allow us to place bounds on certain dimensionless ratios which hold automatically for all systems described by relativistic kinetic theory, regardless of the details of $\mathcal{C}$. To see this, let us first define an inner product on the Hilbert space of functions of momentum,

$$
(g, h) \equiv \beta^{2} \sum_{a} \nu_{a} \int_{p}\left(q^{a}\right)^{2} f_{0}^{a}(p)\left[1 \pm f_{0}^{a}(p)\right] g^{a}(\boldsymbol{p}) h^{a}(\boldsymbol{p}),
$$

with $\nu_{a}$ the degeneracy of species $a$ and $\int_{\boldsymbol{p}} \equiv \int\left(d^{3} \boldsymbol{p} /(2 \pi)^{3}\right)$. Basic considerations such as stability ensure that the linearized collision operator $\mathcal{C}$ is a linear, real, symmetric, positive semidefinite operator under this inner product and strictly positive in the channels we consider. In terms of this inner product, the first-order transport coefficients become $[13,21]$

$$
\eta=\frac{1}{15}(\chi, 1), \quad k_{\mu_{\alpha}}=\frac{T}{3}(\chi, 1) .
$$

The enthalpy density and charge susceptibility can be easily obtained as

$$
\epsilon+\mathcal{P}=\frac{T}{3}(1,1), \quad \chi_{\alpha}=T(1,1) .
$$

$\tau_{\pi}, \tau_{j}$ require inserting $f_{1}$ into the left-hand side of Eq. (7) and using the time derivative to find $f_{2}$ at one space-derivative, one time-derivative order. As shown in Ref. [11], the properties of the inner product and of $\mathcal{C}$ then turn the evaluation into the inner product of the first-order departure from equilibrium $\chi$ with itself:

$$
\eta \tau_{\pi}=\frac{\beta}{15}(\chi, \chi)
$$


The same analysis can be applied to $\tau_{j}$ and we find

$$
k_{\mu_{\alpha}} \tau_{j}=\frac{1}{3}(\chi, \chi) .
$$

It is then insightful to consider these dimensionless ratios,

$$
\frac{\tau_{\pi}}{\eta /(\epsilon+\mathcal{P})}=5 \frac{(\chi, \chi)(1,1)}{(\chi, 1)^{2}}, \quad \frac{\tau_{j}}{D_{\alpha}}=3 \frac{(\chi, \chi)(1,1)}{(\chi, 1)^{2}},
$$

which also have the same number of powers of the collision operator $\left(\chi \propto \mathcal{C}^{-1}\right)$ in the numerator as in the denominator. The triangle inequality implies

$$
\frac{\tau_{\pi}}{\eta /(\epsilon+\mathcal{P})} \geq 5, \quad \frac{\tau_{j}}{D_{\alpha}} \geq 3 .
$$

These results apply to any kinetic theory description of these transport coefficients, as long as the enthalpy density or the charge susceptibility are also consistently computed within the kinetic theory. We remark that the $\ell=1,2$ departures from equilibrium contributing to these transport coefficients do not by construction contribute to the $(\ell=0)$ thermodynamical functions $\epsilon+\mathcal{P}$ or $\chi_{\alpha}$.

In contrast, strong-coupling results from the AdS/CFT correspondence in $\mathcal{N}=4$ SYM theory give for $\tau_{\pi}[12]$ and for the relaxation of a $U(1)$ current in SYM theory [25]

$$
\left.\frac{\tau_{\pi}}{\eta /(\epsilon+\mathcal{P})}\right|_{\mathrm{AdS}}=4-2 \ln (2),\left.\quad \frac{\tau_{j}}{D_{U(1)}}\right|_{\mathrm{AdS}}=\frac{\pi}{2} .
$$

In both cases, these strong-coupling results are approximately half the minimum value attainable in kinetic theory. Finite-coupling corrections [26-30] to the first ratio show a modest increase. We also note that our kinetic theory bounds in Eq. (16) can be shown to become in $d$ spatial dimensions, $d+2$ and $d$, respectively. It would be interesting to derive larger-dimension holographic results in comparison.

Second-order relaxation at (almost) NLO.-We now provide results for the second-order relaxation of the shear stress tensor and of the light quark current $j_{q}$ in QCD. In Ref. [14], we have introduced in great detail a linearized collision operator to "(almost) NLO," (Corrections which lie beyond the kinetic theory picture arise at still higher order.) $2 \leftrightarrow 2$ elastic scatterings and effective $1 \leftrightarrow 2$ inelastic scatterings contribute to the LO collision operator, the former taking the lion's share. At NLO, we found all new scattering processes, and corrections to the LO processes, which are suppressed by a single power of the QCD coupling $g$. As we showed in detail, there are only a few such $\mathcal{O}(g)$ subleading effects. First, the rate of soft $2 \leftrightarrow 2$ scattering is modified; this can be described as an additional momentum-diffusion coefficient $\delta \hat{q}$. This modification and an $\mathcal{O}(g)$ correction to the in-medium dispersion also provide an $\mathcal{O}(g)$ shift in the $1 \leftrightarrow 2$ rate. Next, this $1 \leftrightarrow 2$ splitting rate must be corrected wherever one participant becomes "soft" $(p \sim g T)$ or when the opening angle becomes less collinear. And finally, subtractions are needed because of the way the numerical implementation of the LO scattering kernel [13] already resums a small amount of the NLO effects. We were able to give a relatively simple determination of these effects by the use of light-cone techniques fostered by Ref. [31]. Unfortunately, these methods typically keep track of the incoming and outgoing momentum of a particle but lose track of the momentum which it transfers to the other participants. This momentum transfer also affects the
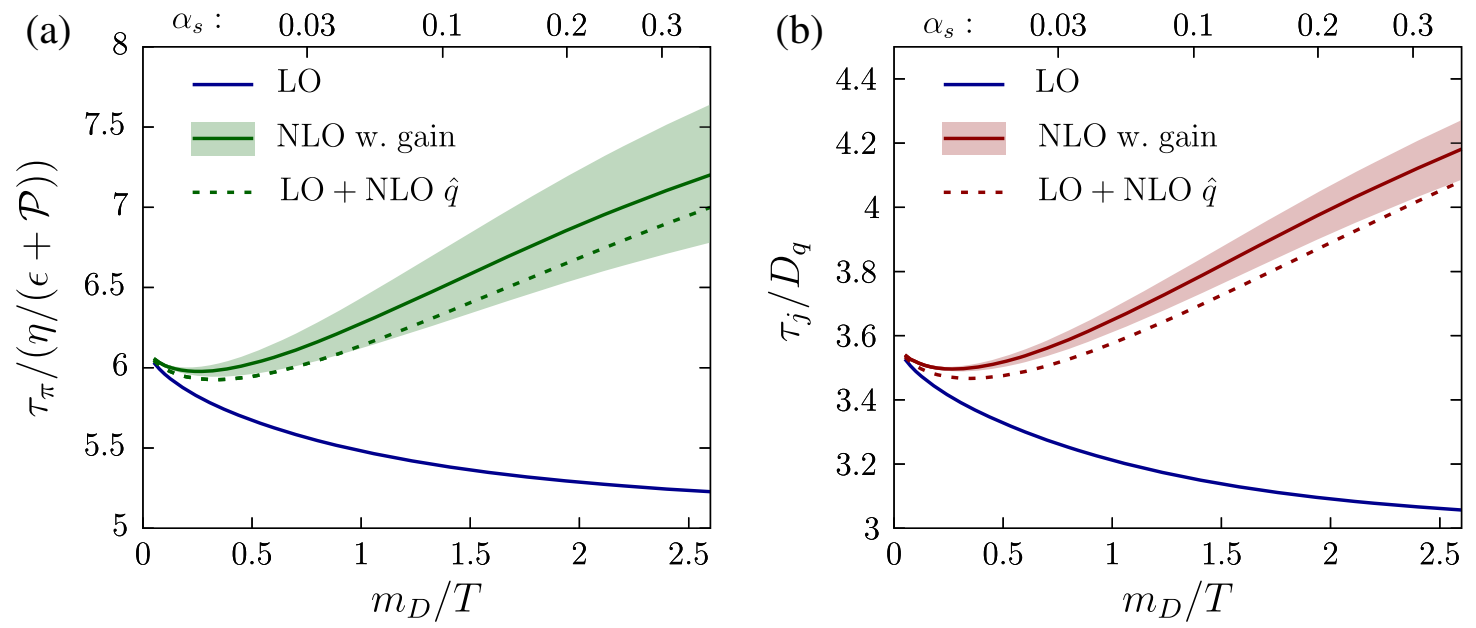

FIG. 2. The second- to first-order ratio of the relaxation coefficients for (a) shear stress $\tau_{\pi} /[\eta /(\epsilon+\mathcal{P})]$ and (b) for quark number diffusion $\tau_{j} / D_{q}$ as a function of $m_{D} / T$ for QCD with three light flavors. (The corresponding value of $\left(\alpha_{s}\right)$ is shown on the upper horizontal axis.) The LO result for $\tau_{\pi}$ is from Ref. [11] and that for $\tau_{j}$ is also new. The uncertainty from the unknown gain terms is shown by the bands; it is estimated as specified in Ref. [14] by the LO value for the gain terms, times $m_{D} / T$, times a constant in the interval $[-2,2]$. The dashed lines represent an estimate in which we include only the NLO $\hat{q}$ to the LO collision operator. 

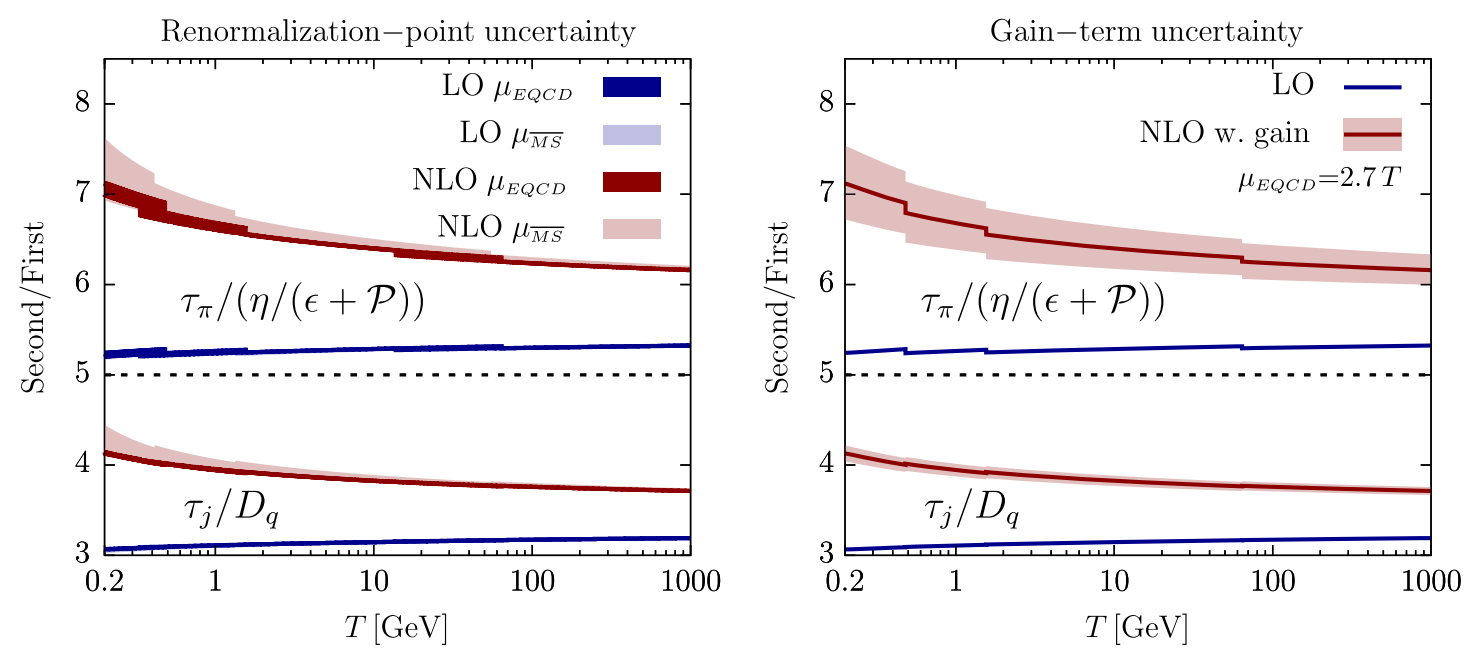

FIG. 3. The second- to first-order ratio of the relaxation coefficients for shear stress $\tau_{\pi} /[\eta /(\epsilon+\mathcal{P})]($ values above 5$)$ and for quark number diffusion $\tau_{j} / D_{q}$ (values below 5) as a function of $T$. On the left, we plot different choices of the running coupling: the solid bands fix the coupling using the two-loop EQCD value with $\mu_{\mathrm{EQCD}}=(2.7 \leftrightarrow 4 \pi) T$, while the shaded bands use the standard $\overline{\mathrm{MS}}$ twoloop coupling with $\mu_{\overline{\mathrm{MS}}}=(\pi \leftrightarrow 4 \pi) T$. On the right, we plot instead in the shaded red bands the estimated uncertainty due to the gain terms. All curves in this plot are obtained using the effective EQCD coupling with $\mu_{\mathrm{EQCD}}=2.7 T$.

departure from equilibrium of the other particle or particles which receive the momentum, generating, in the effective Fokker-Planck approach applicable for these soft scatterings, a gain term. This is an effect which we failed to account for at NLO, hence, the "almost" NLO. However, we estimated that this missing part is most likely small. Finally, we found out that $\eta / s$ and $D_{q}$ at NLO become smaller than their LO counterparts by a factor of 4 at the couplings of relevance for heavy ion collisions; see Fig. 1. The large $\delta \hat{q}$ contribution is by far the main contribution responsible for this behavior.

We now use this (almost) NLO collision operator to determine $\tau_{\pi}$ and $\tau_{j}$ using Eqs. (8) and (15). We solve Eq. (8) with the same variational method as in Ref. [14], which also details the NLO operator $\delta \mathcal{C}$. In Fig. 2, we plot our results for the second-order coefficients $\tau_{\pi}$ and $\tau_{j}$ normalized as in Eq. (15) as functions of the Debye mass $m_{D} \sim g T$ over the temperature. The LO results for $\tau_{\pi}$ were originally obtained in Ref. [11]. Those for $\tau_{j}$ are new and consistent with the leading-log estimate in Ref. [19]. The plot shows that both LO results in solid blue decrease with increasing coupling, approaching the minimum values [Eq. (16)], while the NLO results in solid green and red, respectively, start to differ significantly from the LO at $m_{D} \gtrsim 0.5 T$, where they start growing, getting in the ballpark of $3 / 2$ of the minima when $\alpha_{s} \sim 0.3$. The dashed green or red curves are the results obtained by adding only $\delta \hat{q}$ to the LO collision operator, showing that also in this case it dominates NLO corrections. The bands are obtained by varying the estimate for the unknown gain terms within a range reasonably encompassing their probable size (and sign), as described in Ref. [14]. Intuitively, the LO results approach the bound at increasing coupling because the log-enhanced $2 \leftrightarrow 2$ processes, which force $\chi(p) \propto p$, become less effective at larger couplings, while the other processes drive $\chi(p)$ to a constant, saturating Eq. (15). At NLO, the large $\delta \hat{q}$ drives $\chi(p)$ towards $p^{2}$, which is further from the bound.

Figure 3 presents the more phenomenologically relevant dependence of these second-order coefficients on the temperature. Since only a NNLO treatment would directly include running-coupling effects, this requires that we pick a prescription for relating the running coupling to the temperature. We do so by either using the M $\bar{S}$ coupling in the range $\pi T<\mu_{\overline{\mathrm{MS}}}<4 \pi T$ (leading to the larger, lightshaded bands in the left plot) or via the effective electrostatic QCD (EQCD) coupling with $2.7 T<\mu_{\mathrm{EQCD}}<4 \pi T$ as in Ref. [32] (narrow, dark-shaded bands in the left plot). The discontinuities in the plot occur where we change prescriptions for the number of light fermion species. The right plot in the figure indicates the errors due to the uncertainties from our ignorance of the gain terms which we discussed above.

Conclusions.-Viscous hydrodynamical studies of heavy ion collisions require second-order hydrodynamical coefficients $\tau_{\pi}, \tau_{j}$ which can be understood as relaxation times towards the first-order behavior. While the hydrodynamic coefficients such as $\eta / s$ and $\tau_{\pi} T$ vary by orders of magnitude as a function of the temperature and differ substantially between LO and NLO calculations (see Fig. 1), we have shown that simple dimensionless ratios, Eq. (15), are remarkably robust, varying at most by $40 \%$ as a function of coupling or temperature and between LO and NLO determinations. Furthermore, and more remarkably, we have shown that in any theory which can be described by kinetic theory of ultrarelativistic particles, these dimensionless ratios 
obey inequalities shown in Eq. (16). These inequalities hold regardless of the details of the collision operators, and they give the hydrodynamics practitioner a simple prescription for how to estimate the relation between first-order and secondorder transport coefficients.

It is also remarkable that the bounds we have found fail by a full factor of 2 when we compared them to the results within strongly coupled theories with holographic duals. We conclude that such strongly coupled theories are very far from having a kinetic description. This provides a useful counterpoint to the frequent unspoken assumption that the QGP should have a kinetic description.

J. G. would like to thank Aleksi Kurkela for useful conversations. G. M. would like to acknowledge support by the Deutsche Forschungsgemeinschaft through Grant No. CRC-TR 211 "Strong-interaction matter under extreme conditions." D. T. would like to acknowledge support by the U.S. Department of Energy through Grant No. DE-FG02-88ER40388.

[1] S. S. Adler et al. (PHENIX Collaboration), Phys. Rev. Lett. 91, 182301 (2003).

[2] J. Adams et al. (STAR Collaboration), Phys. Rev. C 72, 014904 (2005).

[3] K. Aamodt et al. (ALICE Collaboration), Phys. Rev. Lett. 107, 032301 (2011).

[4] S. Chatrchyan et al. (CMS Collaboration), Phys. Lett. B 724, 213 (2013).

[5] G. Aad et al. (ATLAS Collaboration), Phys. Rev. C 90, 024905 (2014).

[6] J. Adam et al. (ALICE Collaboration), Phys. Rev. Lett. 117, 182301 (2016).

[7] U. Heinz and R. Snellings, Annu. Rev. Nucl. Part. Sci. 63, 123 (2013).

[8] C. Gale, S. Jeon, and B. Schenke, Int. J. Mod. Phys. A 28, 1340011 (2013).

[9] W. Israel and J. M. Stewart, Ann. Phys. (N.Y.) 118, 341 (1979).

[10] W. A. Hiscock and L. Lindblom, Phys. Rev. D 31, 725 (1985).

[11] M. A. York and G. D. Moore, Phys. Rev. D 79, 054011 (2009).

[12] R. Baier, P. Romatschke, D. T. Son, A. O. Starinets, and M. A. Stephanov, J. High Energy Phys. 04 (2008) 100.
[13] P. B. Arnold, G. D. Moore, and L. G. Yaffe, J. High Energy Phys. 05 (2003) 051.

[14] J. Ghiglieri, G. D. Moore, and D. Teaney, J. High Energy Phys. 03 (2018) 179.

[15] Reference [11] showed that another second-order coefficient $\lambda_{2}$ in the notation of Ref. [12] obeys $\lambda_{2}=-2 \eta \tau_{\pi}$. This still holds at NLO, so we determine this additional coefficient for free.

[16] Reference [17] shows that $\tau_{\pi}$ should be understood as a Wilson coefficient; in the deep IR it receives formally divergent contributions from hydrodynamical fluctuations (long-time tails), which are small at all physically interesting timescales if $\eta / s>0.2$.

[17] P. Kovtun, G. D. Moore, and P. Romatschke, Phys. Rev. D 84, 025006 (2011).

[18] B. Betz, D. Henkel, and D. H. Rischke, Prog. Part. Nucl. Phys. 62, 556 (2009).

[19] J. Hong and D. Teaney, Phys. Rev. C 82, 044908 (2010).

[20] G. S. Denicol, H. Niemi, E. Molnar, and D. H. Rischke, Phys. Rev. D 85, 114047 (2012); 91, 039902(E) (2015).

[21] P. B. Arnold, G. D. Moore, and L. G. Yaffe, J. High Energy Phys. 11 (2000) 001.

[22] J. Ghiglieri, G. D. Moore, and D. Teaney, J. High Energy Phys. 03 (2016) 095.

[23] We use capital letters for four-vectors, bold lowercase ones for three-vectors, and italic lowercase for the modulus of the latter. We work in the "mostly plus" metric so that $P^{2}=-p_{0}^{2}+p^{2}$. The upper sign is for bosons, and the lower sign is for fermions. The full collision operator is $C_{a}$; the collision operator linearized in the departure from equilibrium is $\mathcal{C}_{a}$.

[24] This notation differs from that in Refs. [13,14,21]. $\mathcal{S}^{a}$ there is $-\mathcal{S}^{a}$ here, and $\chi^{a}(p)$ there equals $-q^{a} \chi^{a}(p)$ here.

[25] Y. Bu, M. Lublinsky, and A. Sharon, J. High Energy Phys. 04 (2016) 136.

[26] A. Buchel, J. T. Liu, and A. O. Starinets, Nucl. Phys. B707, 56 (2005).

[27] P. Benincasa and A. Buchel, J. High Energy Phys. 01 (2006) 103.

[28] A. Buchel, Nucl. Phys. B802, 281 (2008).

[29] A. Buchel, Phys. Lett. B 665, 298 (2008).

[30] A. Buchel and M. Paulos, Nucl. Phys. B805, 59 (2008).

[31] S. Caron-Huot, Phys. Rev. D 79, 065039 (2009).

[32] M. Laine and Y. Schroder, J. High Energy Phys. 03 (2005) 067. 